\title{
Comparative analysis of fatty acid metabolism based on transcriptome sequencing of wild and cultivated Ophiocordyceps sinensis
}

\author{
Han Zhang ${ }^{1}$, Pan Yue ${ }^{1}$, Xinxin Tong ${ }^{1}$, Tinghui Gao $^{1}$, Ting Peng $^{1}$, Jinlin Guo $^{\text {Corresp. } 1}$ \\ ${ }^{1}$ Chengdu University of Traditional Chinese Medicine, Chengdu, China \\ Corresponding Author: Jinlin Guo \\ Email address: guo596@cdutcm.edu.cn
}

Background. Ophiocordyceps sinensis is a species endemic to the alpine and high-altitude areas of the Qinghai-Tibet plateau. Although $O$. sinensis has been cultivated since the past few years, whether cultivated $O$. sinensis can completely replace wild $O$. sinensis remains to be determined.

Methods. To explore the differences of $O$. sinensis grown in varied environments, we conducted morphological and transcriptomic comparisons between wild and cultivated samples who with the same genetic background.

Results. The results of morphological anatomy showed that there were significant differences between wild and cultivated $O$. sinensis, which were caused by different growth environments. Then a total of 9,360 transcripts were identified using Illumina paired-end sequencing. Differential expression analysis revealed that $73.89 \%$ differentially expressed genes (DEGs) were upregulated in 0 . sinensis grown under natural conditions compared with that grown under artificial conditions. Functional enrichment analysis showed that some key DEGs related to fatty acid metabolism, including acyl-CoA dehydrogenase, enoylCoA hydratase, 3-ketoacyl-CoA thiolase, and acetyl-CoA acetyltransferase, were upregulated in wild $O$. sinensis. Furthermore, gas chromatography-mass spectrometry results confirmed that the fatty acid content of wild $O$. sinensis was significantly higher than that of cultivated $O$. sinensis and that unsaturated fatty acids accounted for a larger proportion.

Conclusion. These results provide a theoretical insight to the molecular regulation mechanism that causes differences between wild and cultivated $O$. sinensis and improving artificial breeding. 


\section{Comparative analysis of fatty acid metabolism based on}

\section{2 transcriptome sequencing of wild and cultivated}

\section{Ophiocordyceps sinensis}

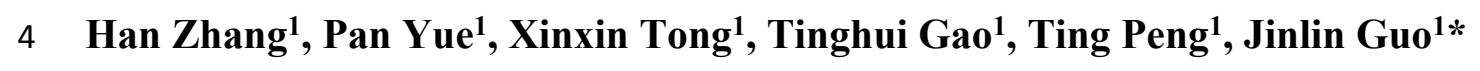

$5{ }^{1}$ Key Laboratory of Standardization of Chinese Medicine, Ministry of Education; Key Laboratory

6 of Systematic Research of Distinctive Chinese Medicine Resources in Southwest China, Resources

7 Breeding Base of Co-founded by Sichuan Province and MOST, Chengdu University of Traditional

8 Chinese Medicine, Chengdu, Sichuan, China

$9 *$ Corresponding Author:

10 Jinlin Guo ${ }^{1}$

111166 Liutai Avenue, Chengdu, Sichuan, 611137, China

12 E-mail address: guo596@cdutcm.edu.cn

\section{Abstract}

Background. Ophiocordyceps sinensis is a species endemic to the alpine and high-altitude areas

of the Qinghai-Tibet plateau. Although O. sinensis has been cultivated since the past few years, whether cultivated $O$. sinensis can completely replace wild $O$. sinensis remains to be determined.

Methods. To explore the differences of $O$. sinensis grown in varied environments, we conducted morphological and transcriptomic comparisons between wild and cultivated samples who with the same genetic background.

Results. The results of morphological anatomy showed that there were significant differences 
22 between wild and cultivated $O$. sinensis, which were caused by different growth environments.

23 Then a total of 9,360 transcripts were identified using Illumina paired-end sequencing. Differential

24 expression analysis revealed that $73.89 \%$ differentially expressed genes (DEGs) were upregulated

25 in $O$. sinensis grown under natural conditions compared with that grown under artificial conditions.

26 Functional enrichment analysis showed that some key DEGs related to fatty acid metabolism,

27 including acyl-CoA dehydrogenase, enoyl-CoA hydratase, 3-ketoacyl-CoA thiolase, and acetyl-

28 CoA acetyltransferase, were upregulated in wild $O$. sinensis. Furthermore, gas chromatography-

mass spectrometry results confirmed that the fatty acid content of wild $O$. sinensis was significantly higher than that of cultivated $O$. sinensis and that unsaturated fatty acids accounted for a larger proportion.

Conclusion. These results provide a theoretical insight to the molecular regulation mechanism that causes differences between wild and cultivated $O$. sinensis and improving artificial breeding.

Keywords: Ophiocordyceps sinensis, wild-grown, cultivated, transcriptome, fatty acid metabolism, GC-MS

\section{Introduction}

Ophiocordyceps sinensis, a complex of larval corpses (sclerotia) and fungal progeny formed by infecting Hepialidae insect larvae, has been used as a valuable material in traditional Asian medicine for more than 2000 years (Sung et al., 2007; Lo et al., 2013). Modern medical research has proven that $O$. sinensis has multiple pharmacological effects such as immune regulation and antibacterial, anti-tumor, and anti-oxidation effects (Gao, 2014; Liu et al., 2015; Dong et al., 2016). 
In recent years, overharvesting owing to global warming and large market demands have led to the rapid reduction of wild $O$. sinensis populations (Dong et al., 2016). To alleviate the shortage and high price, the development of the artificial cultivation industry of $O$. sinensis has been greatly promoted. Artificial cultivation ensures the consistency of the genetic background, thereby creating a comfortable growth environment for $O$. sinensis, artificially avoiding abiotic stress, and considerably shortening the growth cycle (Liu et al., 2016; Xiao et al., 2018). As a result, cultivated O. sinensis differs from wild $O$. sinensis in tissue morphology, stress resistance, accumulation of certain biologically active ingredients, etc., which may also cause differences in efficacy. Therefore, whether cultivated $O$. sinensis can completely replace wild populations or have the same medicinal value remains to be determined.

Currently, the comparison between wild and cultivated $O$. sinensis predominantly focuses on the detection of components' contents (Zheng-Ming et al., 2016), differences in identification (Yang et al., 2017), and research on the pharmacological effects of certain types of biologically active ingredients (Liping et al.); however, such comparisons do not fundamentally explain the formation mechanism of these differences. With the rapid development of high-throughput technologies, applying omics methods to study differences in biological characteristics has become an effective method (Zhu et al., 2014; Xia et al., 2016, 2017). For instance, using mRNA sequencing data of cultivated and wild tomatoes, wild tomatoes were shown to have better salt tolerance and drought resistance based on the molecular levels (Dai et al., 2017). Another study compared the transcriptomes of wild and cultivated ginseng and found that HMG-CoA synthase, 
64 levels of key enzymes of ginseng are upregulated in wild ginseng, which explains the lower 65 ginsenoside content in cultivated ginseng (Zhen et al., 2015).

66 In this study, we obtained morphological differences related to the developmental phenotype 67 of $O$. sinensis by morphological comparison. On the other hand, focused on the whole genome information of $O$. sinensis by comparing the transcriptome sequencing data of wild and cultivated specimens, mining the key genes that led to differences, and finally verifying the results using quantitative real-time polymerase chain reaction (qRT-PCR) and gas chromatography-mass spectrometry. This research has important theoretical significance and application value for perfecting the molecular regulation mechanism that causes differences between wild and cultivated O. sinensis and improving artificial breeding.

\section{Materials \& Methods}

\section{Specimen collection}

Naturally growing $O$. sinensis were harvested at Aba Prefecture, Sichuan Province, China (4500 m, N31 $\left.08^{\prime} 51.9^{\prime \prime}, \mathrm{E} 102^{\circ} 21^{\prime} 26.78^{\prime \prime}\right)$ in May. Cultivated samples were collected from the artificial cultivation workshop at Chengdu Eastern Sunshine Co. Ltd. The genetic background of Hepialidae larvae and $H$. sinensis used in the cultivation is consistent with the wild genetic background. Cultured conditions: one month after the artificial infestation has successfully formed sclerotium, keeping light for 10 hours a day, air humidity above $80 \%$, soil humidity above $20 \%$, temperature $18^{\circ} \mathrm{C}$, and adding nutrients to the soil according to the proportion of the large elements of MS medium. All samples were stored at $-80^{\circ} \mathrm{C}$ until further processing. 


\section{Morphological comparison}

To identify morphological differences between the samples, we first compared the appearance of wild and cultivated $O$. sinensis and recorded photographs. Subsequently, crosscutting was performed at the top and tail of the worm body and the fruiting body at $1 \mathrm{~cm}$, including the middle part, and photographed using Toup View imaging system. Finally, microscopic analysis was performed to further observe the microstructure characteristics, (Jie-Pin, 2006). Paraffin sections were prepared and observed under an electron microscope. Three replicates were conducted for each set of samples.

\section{Transcriptome data acquisition}

Total RNA was extracted using Eastep Super Total RNA Extraction Kit (Promega Shanghai) according to the manufacturer's instructions. After assessing the RNA quality, $1 \mu \mathrm{g}$ of total RNA from each group was used as input material for RNA sample preparation using the NEBNext ${ }^{\mathrm{R}}$ Ultra $^{\mathrm{TM}}$ Directional RNA Library Prep Kit for Illumina ${ }^{\mathrm{R}}$ (NEB, USA) to construct cDNA libraries. Pair-end sequencing was performed using the Illumina Hiseq2000 platform. A total of 100-base paired-end clean reads were generated after removing low-quality reads, adaptor-polluted reads, and reads with high levels of an unknown base. Two biological replicates were performed for RNA-seq.

\section{Gene expression analysis}

Raw data were preprocessed using the fastp tool (http://github.com/openGene/fastp) to obtain clean reads. The Tophat2 aligner tool (http://ccb.jhu.edu/software/tophat/index.shtml) was then used to align sequencing reads to the reference sequences (Daehwan et al., 2013). To eliminate the 
106 effect of different sequencing discrepancies and gene lengths on the gene expression calculation,

107 fragments per kilobases of exon per million fragments mapped (FPKM) values were used to 108 normalize the expression level (Robinson and Oshlack, 2010). To infer the transcriptional changes 109 in both groups, differential expression analysis was performed using the DESeq2 R package 110 according to the express count matrix

111 (https://bioconductor.org/packages/release/bioc/html/DESeq2.html) (Love et al., 2014). A false

112 discovery rate $(\mathrm{FDR}) \leqslant 0.01$ and $\mid \log 2$ (fold change, FC) $\mid \geq 2$ were set as thresholds for the selection 113 of differentially expressed genes (DEGs).

\section{Functional annotation and enrichment of DEGs}

115 All DEGs were annotated using five databases, including NCBI nonredundant protein database, 116 Kyoto Encyclopedia of Genes and Genomes (KEGG), Universal Protein, and Cluster of 117 Orthologous Groups of proteins (COG). Gene ontology (GO) annotations were classified into three ontology categories: molecular function, cellular component, and biological process (Ashburner et al., 2000). KEGG enrichment analysis of the DEGs was performed using the KOBAS software (http://kobas.cbi.pku.edu.cn/kobas3/) (Minoru \& Susumu, 2000) and showed the top 20 pathways with the smallest significant Q value.

qRT-PCR

qRT-PCR was performed on the CFX ConnectTM Optics Module using 2X Ultra SYBR Mixture

124 (TransGen, Beijing, China) according to the manufacturer's instructions. The $\beta$-actin gene was used as a reference to normalize the expression data, and the $2^{-\Delta \Delta C T}$ values were shown as relative expression levels (Thomas, Kenneth \& Livak, 2008). All primers used for the assessment of 
127

128

130

131

132

transcript levels are listed in Table S1.

\section{Fatty acid extraction and identification using GC-MS}

We collected three batches of wild and cultivated $O$. sinensis specimens for fatty acid testing (Table S2). The samples were prepared dried at $60^{\circ} \mathrm{C}$ and grounded to obtain $80 \sim 100 \mathrm{mg}$ of powder. $2 \mathrm{~mL} 5 \%$ hydrochloric acid solution-methanol $(1: 1, \mathrm{~V} / \mathrm{V})$ and $3 \mathrm{~mL}$ chloroformmethanol were added, then water bathed at $85^{\circ} \mathrm{C}$ for $1 \mathrm{~h}$. Next, when cooled to room temperature, $1 \mathrm{~mL}$ of $\mathrm{n}$-hexane was mixed and extracted for $2 \mathrm{~min} .100 \mu \mathrm{L}$ of the supernatant was taken into a $1 \mathrm{~mL}$ volumetric flask and n-hexane was used to make the volume constant. Finally, the fatty acid preparation sample was obtained by filtration through a $0.45 \mu \mathrm{m}$ microporous membrane (Zhihui et al., 2017). All the extracts were analyzed using an Agilent 7890B/5977B gas chromatograph. A TG-5MS capillary column $(30 \mathrm{~m} \times 0.25 \mathrm{~mm} \times 0.25 \mu \mathrm{m})$ was used with helium $(99.999 \%)$ as the carrier gas at a constant flow rate of $1.2 \mathrm{~mL} \mathrm{~min}^{-1}$. The sample $(0.1 \mu \mathrm{L})$ was injected in the splitless mode, and the inlet temperature was $290^{\circ} \mathrm{C}$. The oven temperature program was as follows: the initial temperature was maintained at $80^{\circ} \mathrm{C}$ for $1 \mathrm{~min}$, increased to $200^{\circ} \mathrm{C}$ at the rate of $10^{\circ} \mathrm{C} / \mathrm{min}$, increased from $200^{\circ} \mathrm{C}$ to $250^{\circ} \mathrm{C}$ at the rate of $10^{\circ} \mathrm{C} / \mathrm{min}$, and maintained at $250^{\circ} \mathrm{C}$ for 3 min. Finally, the temperature was increased to $270^{\circ} \mathrm{C}$ at the rate of $2^{\circ} \mathrm{C} / \mathrm{min}$. The qualitative and quantitative analysis of fatty acids was performed according to the GC-MS NIST Mass Spectral Library and the chromatogram of mixed acid fatty standard (NU-CHEK, USA, M20-D, 25mg).

\section{Data availability}

The $O$. sinensis reference genome was derived from the Ensembl Fungi database, ASM44836v1(http://fungi.ensembl.org/Ophiocordyceps sinensis co18 gca 000448365/Info/In 
$148 \mathrm{dex})$. The raw data of wild $O$. sinensis were deposited in the Genome Sequence Archive under the 149 accession number PRJCA000970, and the data of cultivated $O$. sinensis are available at the NCBI 150 Sequence Read Archive under accession numbers SRR5282577 and SRR5282578.

Results

\section{Morphological comparison between wild and cultivated 0 . sinensis}

To perform a preliminary comparison between wild and cultivated $O$. sinensis, the physical characteristics, cross-sectional characteristics of each part, and microscopic observations of

paraffin sections were evaluated to determine the morphological differences associated with the developmental phenotype of $O$. sinensis. The structural characteristics of wild and cultivated $O$. sinensis are consistent with the description in the Chinese Pharmacopoeia: "The insect body is connected to the fungus seat, the surface is dark yellow to yellowish brown, and eight pairs of feet" (Figure S1), and the main difference is reflected in the morphology. The cross-section and microscopic results of each part are shown in Figure 1, S2, and S3. Compared with cultivated $O$. sinensis, the outer wall structure is thinner, and the digestive tract and other organs in the body are less complete. In addition, the inner hyphae are arranged in parallel in the fruiting body, the hyphae in the wild specimens appear to be arranged more closely, and the density is significantly higher than that in cultivated $O$. sinensis specimens. Such differences are mainly caused by their physiological functions and growth environment. sinensis 
169

170

171

172

173

174

After performing quality control of the sequencing data, a total of $28.85 \mathrm{~Gb}$ of clean data was obtained, and the percentage of Q30 bases was $\geq 93.68 \%$ (Table S3), indicating that the higher the accuracy of base identification during the sequencing process, the sequence quality can be used for subsequent analysis. The efficiency of comparison with the reference genome was between 81.00\% and 84.40\% (Table S4), and 9360 transcripts were obtained after mapping, including 997 newly predicted genes.

DEGs were calculated based on FPKM, with an FDR of $<0.01$ and $\mid \log 2$ (fold change, FC) $\mid \geq 2$. A total of 563 DEGs in the wild and cultivated $O$. sinensis were identified. Compared with the wild group, the expression of 415 genes was downregulated and the expression of only 148 genes was upregulated in the cultivated group. The number of DEGs in the wild group was significantly higher (Figure 2).

\section{Functional enrichment analysis of DEGs}

Overall, 563 DEGs were annotated by Blast2GO to a total of 274 annotation entries. The GO term cellular component was mainly enriched into 14 terms, including membrane, organelle, macromolecular complex, etc. In the GO term molecular function, a total of 225 DEGs were enriched into 8 terms, mainly catalytic activity, binding, transcription regulator activity and transporter activity, etc. In biological process, 193 DEGs were significantly enriched in 14 terms, mainly metabolic process, cellular process, and single-organism process (Figure 3A). KEGG annotation and enrichment results are shown in Table S5 and Figure 3B. Fatty acid degradation, fatty acid metabolism, butanoate metabolism, and $\beta$-alanine metabolism showed the most significant differences in the metabolic pathways, of which the most abundant was biosynthesis of 
190

191

192

193

194

195

196

197

198

199

200

201

202

203

204

205

206

207

208

209

210

antibiotics.

Based on these results, we found that fatty acid metabolism was the most significant difference between wild and cultivated $O$. sinensis. On comparing these data with major functional databases, the DEGs in the fatty acid pathway were identified and were found to code for acylCoA dehydrogenase (ACAD) (OCS_02728), enoyl-CoA hydratase (ECH) (OCS_06591), 3ketoacyl-CoA thiolase (KAT) (OCS_04932), and acetyl-CoA acetyltransferase (ACAT) (OCS_01242/OCS_05099).

\section{Fatty acid metabolism-related DEGs involved in wild and cultivated 0 . sinensis}

Based on the results of functional enrichment analysis of DEGs, we selected fatty acids as the focus of research on the differences between wild and cultivated $O$. sinensis. Acetyl-CoA (Ac$\mathrm{CoA})$ is the precursor of the Malongl-CoA (MalCoA) in fungi and is the final product of fatty acid degradation. Under the catalysis of fatty acid synthase (FANS) and other enzymes, one molecule of MalACP is added in each step, increasing the length of two carbon atoms each time; after seven additions, hexadecanoyl-[acp] is formed and, finally, palmitic acid is formed under the action of FASN (Figure 4A) (Nowinski et al., 2018). In addition, hexadecanoyl-CoA can be used as a substrate for the synthesis of longer-chain fatty acids, generating different types of fatty acids under the catalysis of different enzymes.

In this study, the enzymes encoded by the DEGs of the significantly enriched metabolic pathways participated in the $O$. sinensis fatty acid $\beta$-oxidation pathway, including four steps: dehydrogenation, hydration, re-dehydrogenation, and thiolysis (Shen \& Burger, 2009). ACAD, $\mathrm{ECH}$, and KAT were significantly downregulated in cultivated $O$. sinensis. ACAD participates in 
211 the first reaction of fatty acid and amino acid $\beta$-oxidation under the action of flavin adenine

212 dinucleotide cofactor (Kim \& Miura, 2004). ECH catalyzes the second step of fatty acid $\beta$ -

213 oxidation, promoting the cis-addition of water molecules on the trans-2-enoyl-CoA thioester

214 double bond to form $\beta$-hydroxyacyl-CoA thioester (Willadsen \& Eggerer, 2008). KAT catalyzes

215 the final step of fatty acid $\beta$-oxidation to produce one molecule of Ac-CoA and one molecule of

216 acyl-CoA (Mano et al., 1996). The $\beta$-oxidation that occurs in mitochondria and peroxisomes is the

217 most important form of oxidation and provides a large amount of energy for organisms by coupling

218 with the tricarboxylic acid cycle and electronic respiratory chain (Neely and Morgan, 1974). The

219 results showed that the genes encoding various enzymes involved in the degradation and synthesis

220 of fatty acids were downregulated in cultivated $O$. sinensis, which indicates that fatty acid

221 metabolism is more active in wild $O$. sinensis (Figure 4B). Moreover, the product of palmitic acid

222 decomposition is Ac-CoA. When fatty acid metabolism is weakened, the downstream citric acid

223 cycle/tricarboxylic acid cycle, alanine aspartate metabolism, and butyrate metabolism also

224 weakens (Figure 4A). Because of the long growth cycle of wild $O$. sinensis and the lack of nutrients

225 in the living environment, we speculate that $O$. sinensis relies mainly on its own fatty acid oxidative

226 decomposition to provide energy for itself, and on $\beta$-oxidation, especially via palmitic acid

227 decomposition.

228 Validation of transcriptome data by qRT-RCR

229 To confirm the reliability of the sequencing data, 12 DEGs were randomly selected to validate

the RNA-Seq expression profiles. As expected, qRT-PCR results showed that most of the mRNAs 
232 were downregulated in the cultivated O. sinensis (Figure 5) and those of two genes were

233 upregulated, indicating that our data were accurate and reliable in the subsequent analyses.

\section{Quantitative analysis of fatty acid content}

235 GC-MS was performed to analyze the fatty acids of $O$. sinensis. The results showed that the

236 types of fatty acids in the wild and cultivated specimens were basically the same (Figure 6) but

237 that the content was quite different. The content of fatty acids in the wild group was much higher

238 than that in the cultivated group, which is consistent with the previously reported (Yang et al.

239 2009). Furthermore, among all types of fatty acids, oleic acid (C18:1n9c), linoleic acid (C18:2n6c),

240 and palmitic acid (C16:0) are the most important components and account for up to 89.38\%-

$24194.88 \%$. From the perspective of fatty acid types, the content of unsaturated fatty acids in $O$.

242 sinensis was higher than the content of saturated fatty acids. In addition, the content of fatty acids

243 in $O$. sinensis has a certain aging effect. With the extension of storage life, the content of fatty

244 acids in medicinal materials is continuously reduced (Table S6).

Discussion

247 It is well known that $O$. sinensis is a typical entomogenous fungus. When Hirsutella sinensis 248 successfully infects the host Hepialus larvae, its bud tube breaks to form a long spindle-shaped 249 fungus, which proliferates and differentiates in an apical budding manner and matches with each other. The phytoplasma grows hyphae and gradually fills the entire body to form sclerotia (Zhang et al., 2011, 2012; Guo et al., 2015). Compared with $O$. sinensis growing in a comfortable artificial 
253 rely on the insect body itself, which causes a greater degree of decomposition in an insect body 254 part and a looser texture.

255 In the Qinghai-Tibet Plateau, which is subject to low temperatures and strong ultraviolet rays,

256 the main vegetation type is the alpine meadow area, and the texture is sandy loam and harder soils

257 (Zhou et al., 2018). These environmental factors directly affect the formation of sclerotia and

258 fruiting bodies of $O$. sinensis. For instance, under natural conditions, the sclerotium needs to

259 overcome the strong resistance of the soil when it grows out of the pedestal; thus, the sclerotium

260 is stronger and tougher than that growing in good conditions and has a tighter organization. In

261 addition, the length and intensity of light received by wild $O$. sinensis is greater, resulting in slower

262 growth but stronger fruiting bodies (larger diameter stroma). In contrast, the fruiting bodies grow

263 rapidly and slender without this condition, similar to cultivated $O$. sinensis (Tu et al., 2010; Tong

264 et al., 2020).

265 In this study, the genetic background of wild and cultivated $O$. sinensis was identical, but there was a large difference in the growth environment. Because the wild $O$. sinensis need to resist the harsh environment of the plateau, the DEGs identified in this study may play an important role in the process of growth and metabolism, differences in substance accumulation, and response to abiotic stress. Studies have reported that the types and contents of chemical components are not much different between wild and cultivated O. sinensis (Hongxia, Xinhua \& Wei, 2012; Liu et al., 2016; Zheng-Ming et al., 2016). However, the palmitic acid and oleic acid content of the fatty acids, which are the main components, is much higher in wild than in cultivated $O$. sinensis (Yang et al., 2009). This conclusion is consistent with our transcriptome data analysis results, which can 
274 be an indicator to distinguish between wild and cultivated $O$. sinensis.

275 At present, research on fatty acids in $O$. sinensis mainly focuses on detection methods and 276 composition comparisons (Yuan-Can et al., 2015), and only few studies have assessed the 277 physiological functions. It was proved that high cold hardiness plants have a higher proportion of

278

unsaturated fatty acids, and a lower phase transition temperature can maintain the fluidity of the membrane at low temperatures to maintain normal physiological functions (Šajbidor, 1997).

Research on the cold resistance of winter wheat found that when the temperature decreases, the content of unsaturated linolenic acid increases and that of palmitic acid decreases (Dong-Wei et al., 2013), indicating that these two fatty acids play the greatest role in the cold resistance of winter wheat. Therefore, it is speculated that linolenic acid and palmitic acid can be used as indicator fatty acids for cold resistance in winter wheat. In fact, wild O. sinensis is subject to long-term lowtemperature stress, which may be attributed to a significantly higher content of unsaturated fatty acids than cultivated $O$. sinensis.

\section{Conclusion}

In summary, a comparative morphological and transcriptomic analysis of $O$. sinensis grown in varied environment was performed. The present results show that natural and cultivated environments will cause significant differences in the morphology. Reflected in the high density of wild $O$. sinensis fruiting body hyphae and higher digestibility of the worm body. According to the transcriptome, genes involved in fatty acid metabolism were upregulated including ACAD, ECH, KAT and ACAT, which conformed that fatty acids are higher in wild O. sinensis at the 
295

296

297

298

299

300

301

302

303

304

305

306

307

308

309

310

311

312

313

314

315

molecular level. Furthermore, This research has important theoretical significance and application

value for perfecting the molecular regulation mechanism that causes differences between wild and cultivated $O$. sinensis and improving artificial breeding.

\section{Conflicts of Interest}

The authors declare no conflict of interest.

\section{Acknowledgements}

This study was supported by the Natural Sciences Foundation of China Science (Nos. 81872959, 81373920, 30801522). Sichuan Province Youth Innovation Team Fund (No. 19CXTD0055).

\section{References}

Ashburner M, Ball CA, Blake JA, Botstein D, Cherry JM. 2000. Gene ontology: Tool for the unification of biology. Nature Genetics 25:25-29.

Daehwan, Kim, Geo, Pertea, Cole, Trapnell, Harold, Pimentel, Ryan, Kelley. TopHat2: accurate alignment of transcriptomes in the presence of insertions, deletions and gene fusions. Genome Biol 14:R34

Dai Q, Geng L, Lu M, Jin W, Nan X, He PA, Yao Y. 2017. Comparative transcriptome analysis of the different tissues between the cultivated and wild tomato. PLOS ONE 12:1-18. DOI: 10.1371/journal.pone.0172411.

Dong-Wei X, Xiao-Nan W, Lian-Shuang FU, Jian S, Tao G, Zhuo-Fu LI. 2013. Effects of Low Temperature Stress on Membrane Fatty Acid in Tillering Node of Winter Wheat. Journal of 
Triticeae Crops 33:746-751.

317

318

319

320

321

322

323

324

325

326

327

328

329

330

331

332

333

334

335

336

Dong CH, Wen-Jia LI, Zeng-Zhi LI, Yan WJ, Tai-Hui LI, Liu XZ. 2016. Cordyceps industry in China: current status, challenges and perspectives__ Jinhu declaration for cordyceps industry development. Mycosystema 35:1-15.

Gao C. 2014. Advances in research of the artificial cultivation of Ophiocordyceps sinensis in China. Critical Reviews in Biotechnology 3:233.

Guo LX, Xu XM, Liang FR, Yuan JP, Peng J, Wu CF, Wang JH. 2015. Morphological observations and fatty acid composition of indoor-cultivated cordyceps sinensis at a highaltitude laboratory on Sejila Mountain, Tibet. PLOS ONE 10. DOI: 10.1371/journal.pone.0126095.

Hongxia Z, Xinhua G, Wei C. 2012. Levels of Selected Chemical Components in Cordyceps sobolifera Fruit Bodies Cultivated Artificially and Collected from the Wild. Acta Edulis Fungi 19:59-62.

Jie-Pin Y. 2006. Improvement of traditional paraffin section preparation methods. Journal of Biology 45-46.

Kim JJ, Miura R. 2004. Acyl-CoA dehydrogenases and acyl-CoA oxidases. Structural basis for mechanistic similarities and differences. 271:483-493.

Liping, Luo, Gulibahaer, Shadier, Peter, Schmidtke, Claudius, U., Meyer, Waike. Cordycepin is an Immunoregulatory Active Ingredient of Cordyceps sinensis 36:967-980.

Liu Y, Wang J, Wang W, Zhang H, Zhang X, Han C. 2015. The Chemical Constituents and Pharmacological Actions of Cordyceps sinensis. Evidence-based Complementary \& 
Alternative Medicine (eCAM) 2015.

338

339

340

341

342

343

344

345

346

347

348

349

350

351

352

353

354

355

356

357

Liu F, Wu XL, Liu Y, Chen DX, Yang DJ. 2016. [Progress on molecular biology of Isaria farinosa, pathogen of host of Ophiocordyceps sinensis during the artificial culture].

Zhongguo Zhong yao za zhi = Zhongguo zhongyao zazhi $=$ China journal of Chinese materia medica 41:403-409.

Lo HC, Hsieh C, Lin FY, Hsu TH. 2013. A Systematic Review of the Mysterious Caterpillar Fungus Ophiocordyceps sinensis in Dong-ChongXiaCao ( Dōng Chóng Xià Căo) and Related Bioactive Ingredients. J Tradit Complement Med 3:16-32.

Love MI, Huber W, Anders S. Moderated estimation of fold change and dispersion for RNA-seq data with DESeq2. Genome Biology 15:550.

Mano S, Hayashi M, Kondo M, Nishimura M. 1996. cDNA cloning and expression of a gene for isocitrate lyase in pumpkin cotyledons. Plant and Cell Physiology 37:941-948. DOI: 10.1093/oxfordjournals.pcp.a029043.

Minoru K, Susumu G. 2000. KEGG: Kyoto Encyclopedia of Genes and Genomes. Nucleic Acids Research:1.

Neely JR, Morgan HE. Relationship Between Carbohydrate and Lipid Metabolism and the Energy Balance of Heart Muscle. 36:413-459.

Nowinski SM, Van Vranken JG, Dove KK, Rutter J. 2018. Impact of Mitochondrial Fatty Acid Synthesis on Mitochondrial Biogenesis. Current Biology 28:R1212-R1219. DOI: 10.1016/j.cub.2018.08.022.

Robinson MD, Oshlack A. A scaling normalization method for differential expression analysis of 
358

359

360

361

362

363

364

365

366

367

368

369

370

371

372

373

374

375

376

377

378

RNA-seq data. Genome Biology 11:R25.

Šajbidor J. 1997. Effect of some environmental factors on the content and composition of microbial membrane lipids. Critical Reviews in Biotechnology 17:87-103.

Shen YQ, Burger G. 2009. Plasticity of a key metabolic pathway in fungi. Functional and Integrative Genomics 9:145-151. DOI: 10.1007/s10142-008-0095-6.

Sung GH, Hywel-Jones NL, Sung JM, Luangsa-Ard JJ, Shrestha B, Spatafora JW. 2007. Phylogenetic classification of Cordyceps and the clavicipitaceous fungi. Studies in Mycology 57:5-59.

Thomas S, Kenneth D, Livak J. 2008. Analyzing real-time PCR data by comparative CT method. Nature Protocols 3:1101-1108.

Tong X, Zhang H, Wang F, Xue Z, Guo J. 2020. Comparative transcriptome analysis revealed genes involved in the fruiting body development of Ophiocordyceps sinensis. PeerJ 8:e8379.

Tu YQ, Zhang DL, Zeng W, Chen SJ, Yin DH. 2010. Effect of environmental factors on the formation of fruiting bodies of Cordyceps sinensis. Edible Fungi of China 29:24-25.

Willadsen P, Eggerer H. 2008. Substrate Stereochemistry of the Enoyl-CoA Hydratase Reaction\&nbsp; Febs Journal 54:247-252.

Xia F, Chen X, Guo MY, Bai XH, Zhou XW. 2016. High-throughput sequencing-based analysis of endogenetic fungal communities inhabiting the Chinese Cordyceps reveals unexpectedly high fungal diversity. Scientific Reports 6:33437.

Xia EH, Yang DR, Jiang JJ, Zhang QJ, Liu Y, Liu YL, Zhang Y, Zhang H Bin, Shi C, Tong Y, 
379

380

381

382

383

384

385

386

387

388

389

390

391

392

393

394

395

396

397

398

399

Kim C, Chen H, Peng YQ, Yu Y, Zhang W, Eichler EE, Gao LZ. 2017. The caterpillar fungus, Ophiocordyceps sinensis, genome provides insights into highland adaptation of fungal pathogenicity. Scientific Reports 7:1-11. DOI: 10.1038/s41598-017-01869-z.

Xiao, Qing, Liu, Wenjia, Quanping, Zhengming, Qian, Xingzhong, Caihong, Dong. 2018. A breakthrough in the artificial cultivation of Chinese cordyceps on a large-scale and its impact on science, the economy, and industry. Critical reviews in biotechnology 39:181191.

Yang FQ, Feng K, Zhao J, Li SP. Analysis of sterols and fatty acids in natural and cultured Cordyceps by one-step derivatization followed with gas chromatography-mass spectrometry. 49:1172-1178.

Yang L, Xiao-Yue W, Zi-Tong G, Jian-Ping H, Li X. 2017. Detection of Ophiocordyceps sinensis and Its Common Adulterates Using Species-Specific Primers. Frontiers in Microbiology 8:1179.

Yuan-Can X, Xiao-Feng C, Qi D, Liang T, Feng-Zu HU. 2015. Qualitative and Quantitative Comparative Analysis of Fatty Acids in Cordyceps sinensis from Different Regions of Yushu Prefecture of Qinghai Province. Journal of Chinese Pharmaceutical ences 50:10981103.

Zhang Y, Li E, Wang C, Li Y, Liu X. 2012. Ophiocordyceps sinensis, the flagship fungus of china: Terminology, life strategy and ecology. Mycology 3:2-10. DOI: $10.1080 / 21501203.2011 .654354$.

Zhang G, Yu J, Wu G, Liu X. 2011. Factors influencing the occurrence of Ophiocordyceps 
400

401

402

403

404

405

406

407

408

409

410

411

412

413

414

415

416

417

sinensis. Shengtai Xuebao/ Acta Ecologica Sinica 31:4117-4125.

Zhen G, Zhang L, Du YN, Yu RB, Liu XM, Cao FR, Chang Q, Deng XW, Xia M, He H. 2015.

De novo assembly and comparative analysis of root transcriptomes from different varieties

of Panax ginseng C. A. Meyer grown in different environments. Science China Life

Sciences 58:1099-1110. DOI: 10.1007/s11427-015-4961-x.

Zheng-Ming Q, Wen-Qing LI, Min-Tian S, Xing-Zhong L, Er-Wei LI, Wen-Jia LI. 2016.

Analysis of chemical compounds in Chinese cordyceps. Mycosystema 87:271-289.

Zhihui B, Ruilian YU, Simin W, Wen J, Jiawei S, Zhen YU, Jinguo XU, Gaohong L. 2017.

Comparative Study on the Contents of Fatty Acids in Isaria cicadae,Cordyceps militaris and Cordyceps sinensis. China Pharmacy 28:4252-4256.

Zhou K, Zhang J, Huang X, Yuan L, Luo Z, Shi L, Wei K. 2018. Suitability and regionalization of Ophiocordyceps sinensis in the Tibetan Plateau. Shengtai Xuebao/ Acta Ecologica Sinica 38:2768-2779. DOI: 10.5846/stxb201704280779.

Zhu Y, He L, Xu X, Chen S, Shi L, Xiang L, Sun W, Li Y, Song J, Luo H, Sun C, Li C. 2014. Transcriptome analysis of the Ophiocordyceps sinensis fruiting body reveals putative genes involved in fruiting body development and cordycepin biosynthesis. Genomics 103:154159. DOI: 10.1016/j.ygeno.2014.01.002. 


\section{Figure 1}

Overview of the structure and morphology of wild-grown and cultivated $O$. sinensis.

(A-B, $\mathrm{H}-\mathrm{I})$, The microscopic characteristics of fruiting body and worm (middle position of structure); (C-D, F-G), The anatomical section characteristics of fruiting body and worm (middle position of structure). $\mathrm{E}$, The characteristics of appearance of $O$. sinensis.
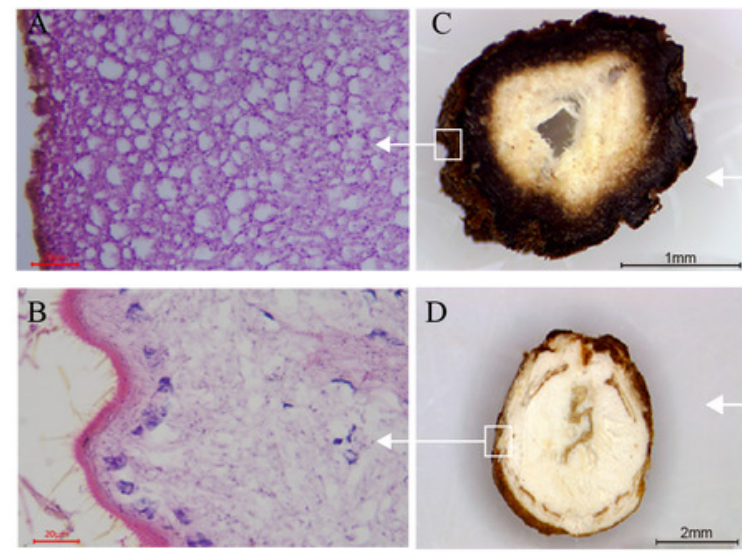

$\vdash----\frac{-----}{\text { wild-grown } O \text {. sinensis }}$
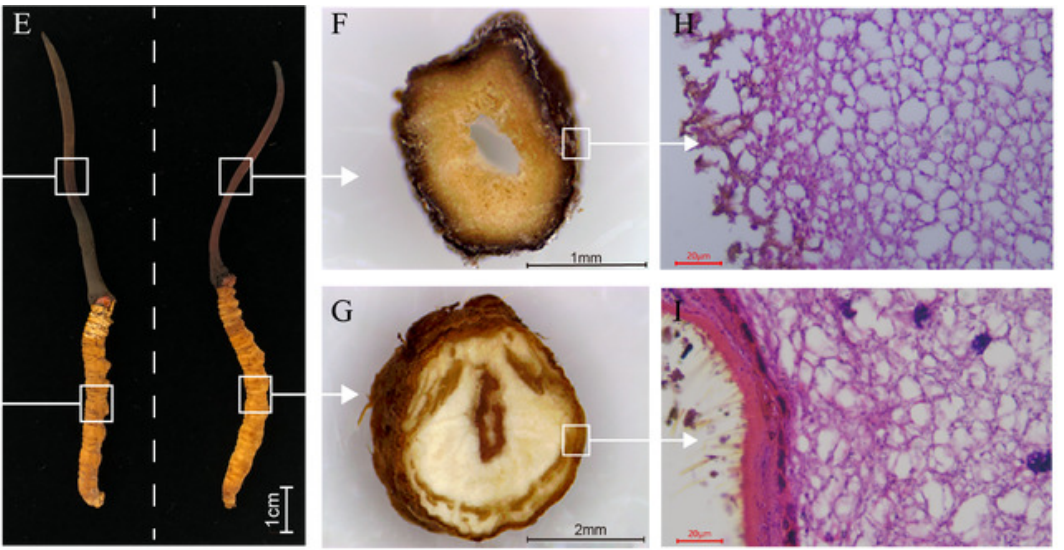

cultivated $O$. sinensis 
Figure 2

Results of differential analysis.

A, Volcano map of differentially expressed genes (wild vs. cultivated). B, Heatmap of differentially expressed genes.

A

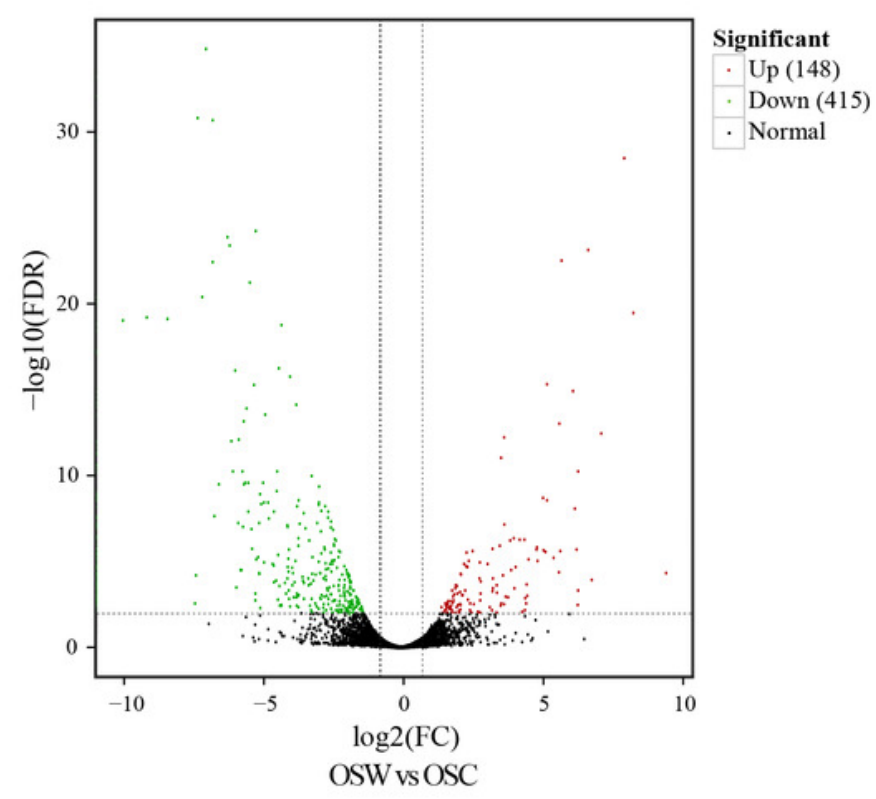

B

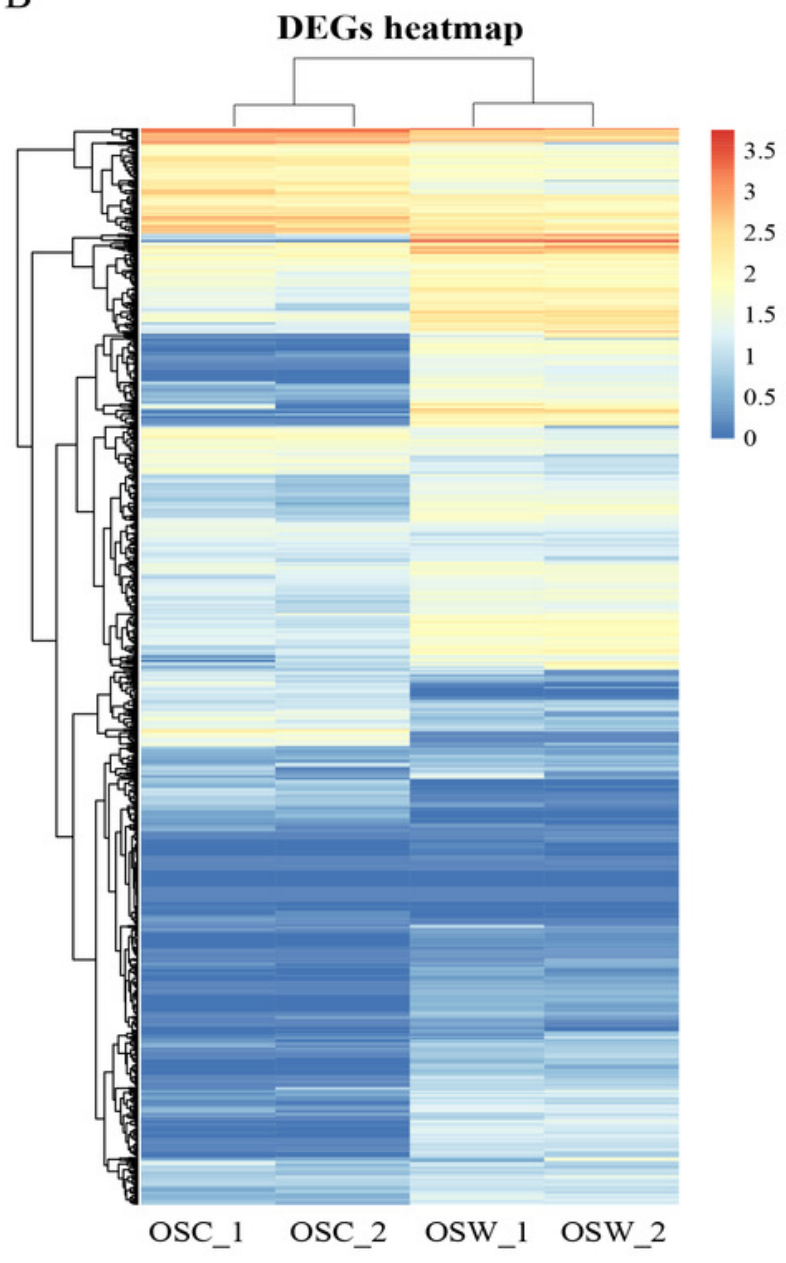


Figure 3

Functional annotation and enrichment results of differentially expressed genes (DEGs)

Functional annotation and enrichment results of differentially expressed genes (DEGs). A.

Gene ontology functional enrichment map of DEGs. B. Analysis of Kyoto Encyclopedia of

Genes and Genomes significant enrichment of DEGs.

A

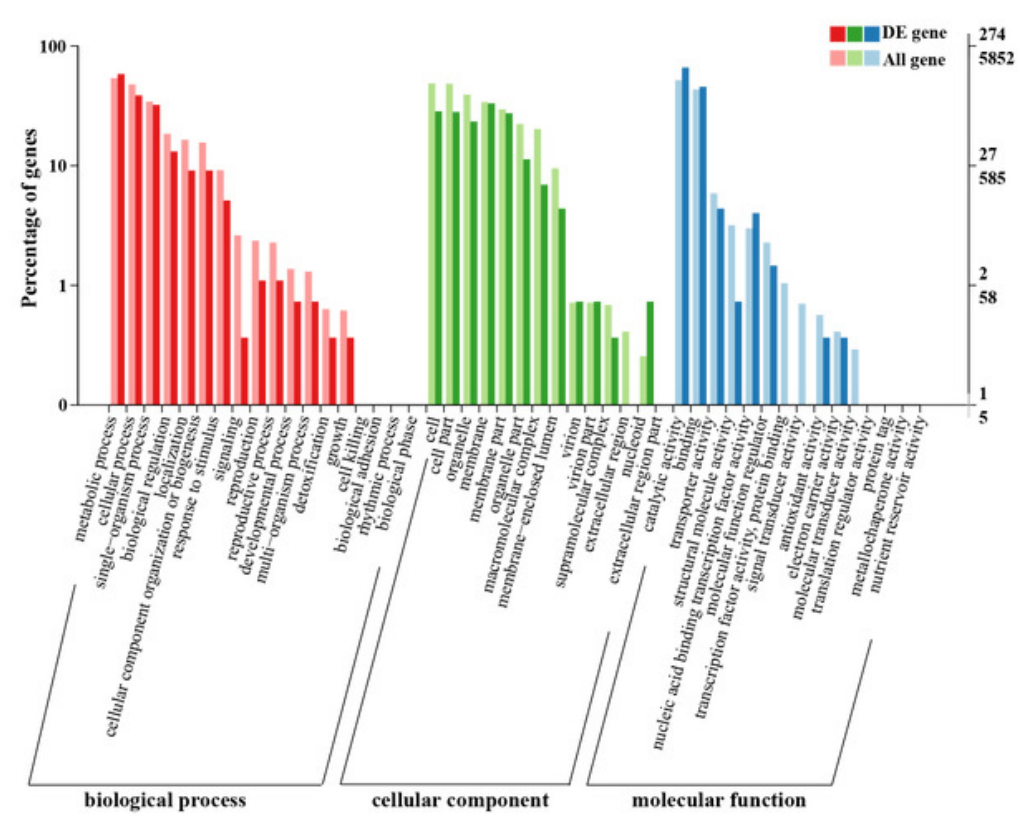

B

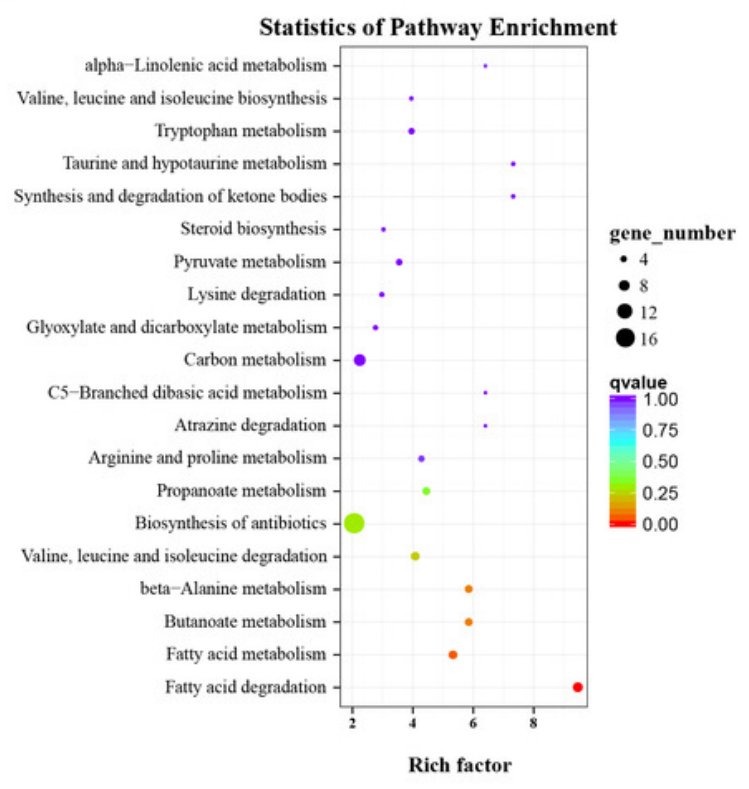




\section{Figure 4}

Metabolism and degradation of palmitic acid in O.sinesis.

A, Degradation of palmitic acid in O.sinesis. (1) Palmitoyl coenzyme A is oxidized and decomposed to generate myristoyl-CoA. (2) Butyryl-CoA is oxidized and decomposed to produce Acetyl-CoA. B, heatmap of lipid-related DEGs, the green marked as a key gene in palmitic acid degradation pathway. *ACSL, long-chain acyl-CoA synthetase. CPT, carnitine 0palmitoyltransferase. ACP, acyl carrier protein,

A

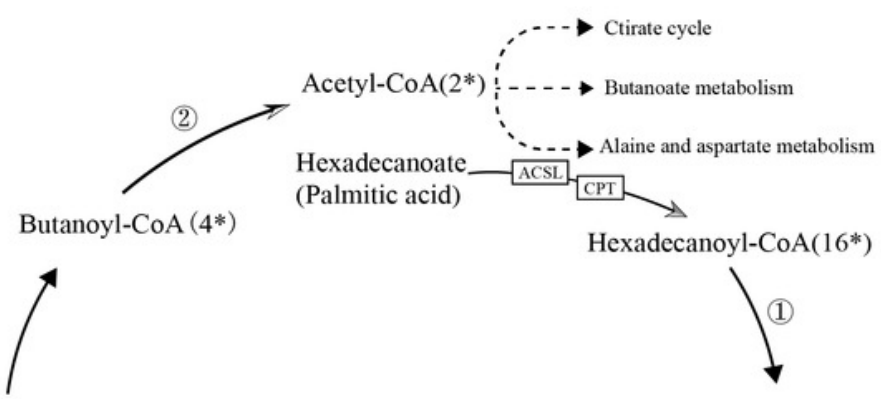

Hexanoyl-CoA(6*)

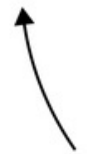

FATTY ACID DEGRADATION Tetradecanoyl-CoA(14*) (beta - Oxidation)

Octanoyl-CoA $\left(8^{*}\right)$
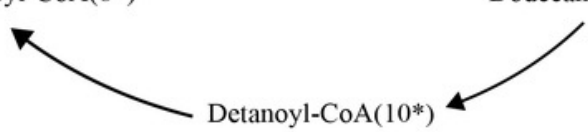

B

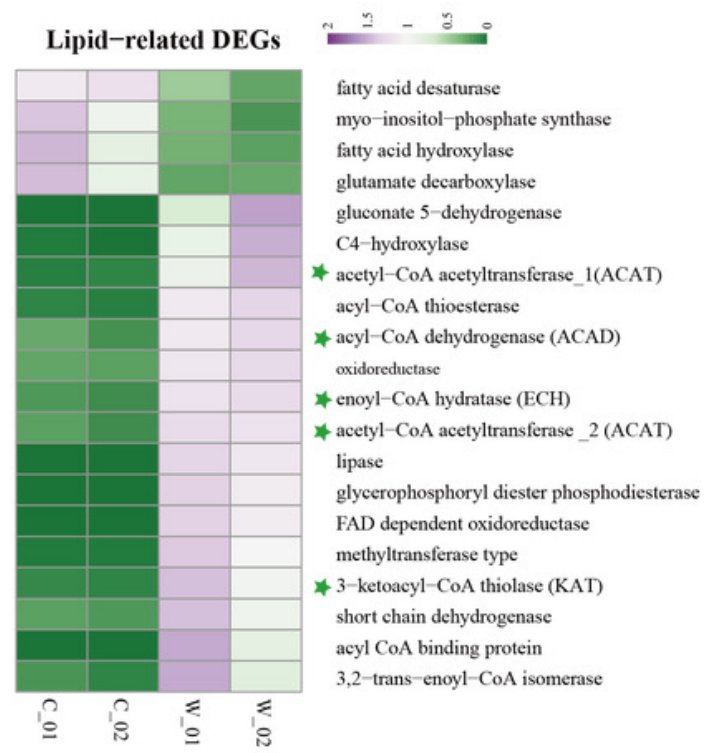

Tetradecanoyl-CoA

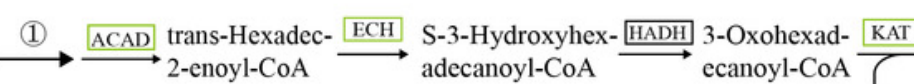

$\stackrel{\text { (2) }}{\longrightarrow} \stackrel{\mathrm{ACAD}}{\longrightarrow} \begin{aligned} & \text { trans-But-2- } \\ & \text { enoyl-CoA }\end{aligned} \stackrel{\text { ECH }}{\longrightarrow} \begin{aligned} & \text { S-3-Hydroxyb- } \\ & \text { butanoyl-CoA }\end{aligned} \stackrel{\text { HADH }}{\longrightarrow}$ Acetoacetyl-CoA $\stackrel{\text { ACAT }}{\longrightarrow}$ Acetyl-CoA

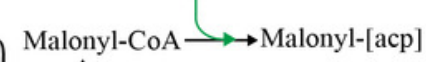

Fatty acid biosynthesis (fungi) 
Figure 5

qRT-PCR verification of the expressed genes in Illumina sequencing

The expression value of $\beta$-actin was used as an inner control, the wild as a control compared to cultivated. All data are means of 3 replicates with error bars indicating SD.

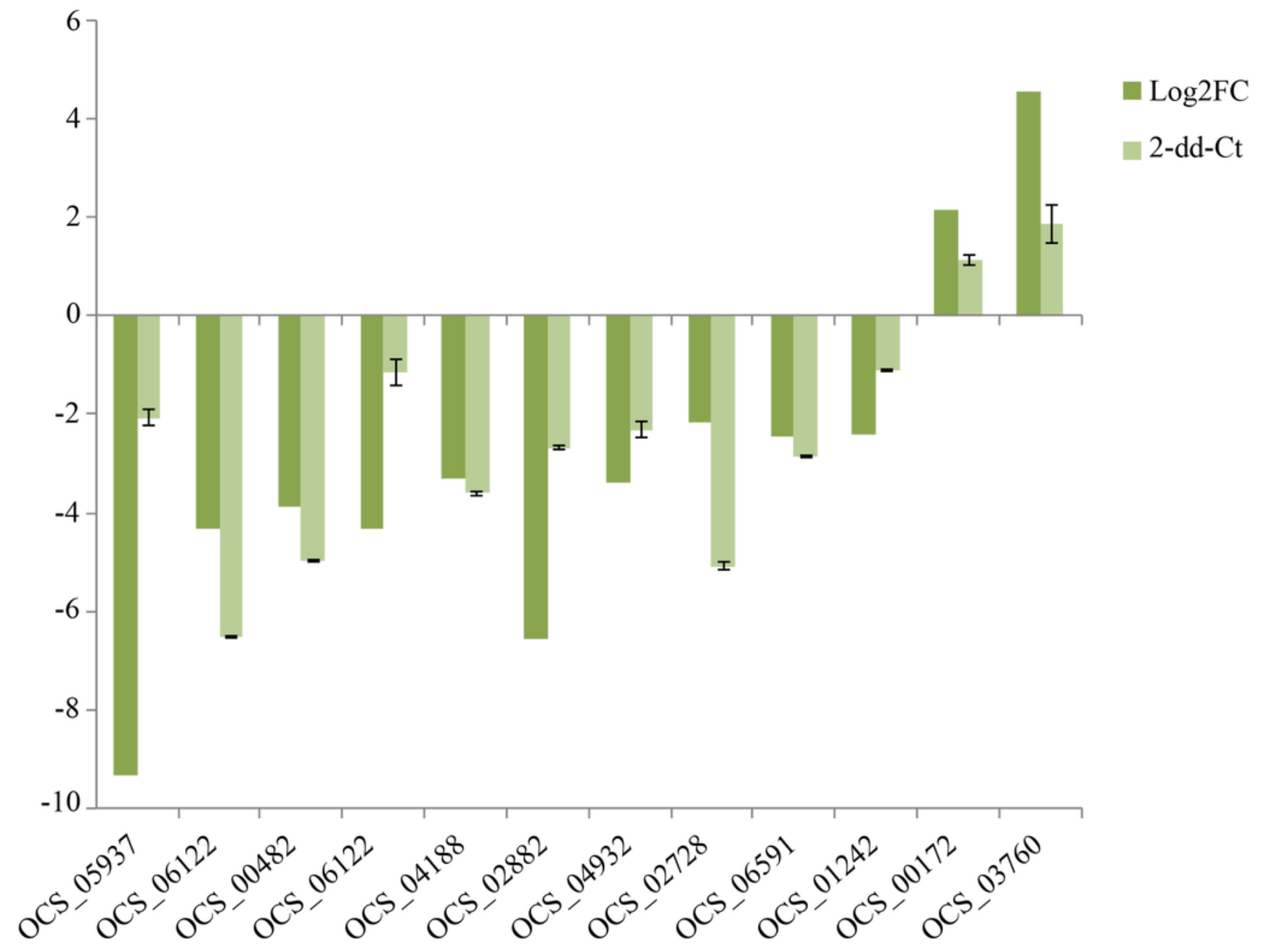




\section{Figure 6}

The ion chromatograms of fatty acid

A. Total ion chromatograms of mixed fatty acid methyl ester standard. B. The ion chromatograms of $O$. sinensis (OSW_S1). 1-Methyl dodecanoate (C12:0); 2-Methyl myristoleate (C14:1n5); 3-Methyl myristate (C14:0); 4-Methyl palmitate (C16:0); 5-Methyl heptadecanoate (C17:0); 6-Methyl linoleate (C18:2n6c); 7-Methyl oleate (C18:1n9c); 8-Methyl stearate (C18:0); 9-Methyl arachidate (C20:0); 10-Methyl dodecanoate (C22:0); and 11Methyl tricosanoate (C23:0). Red numbers represent the content $(\mathrm{mg} / \mathrm{g})$ in the wild group, blue numbers represent the content in the cultivated group, "-" indicates content below the detection limit. 
A

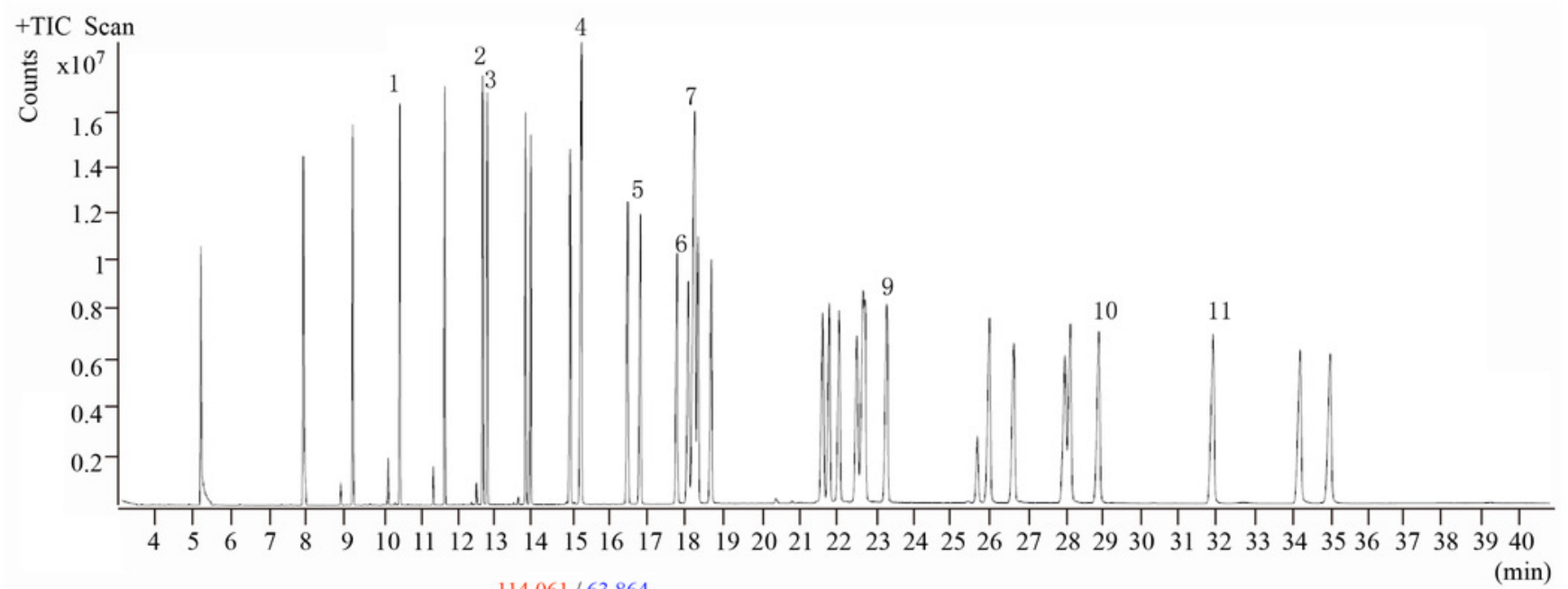

B

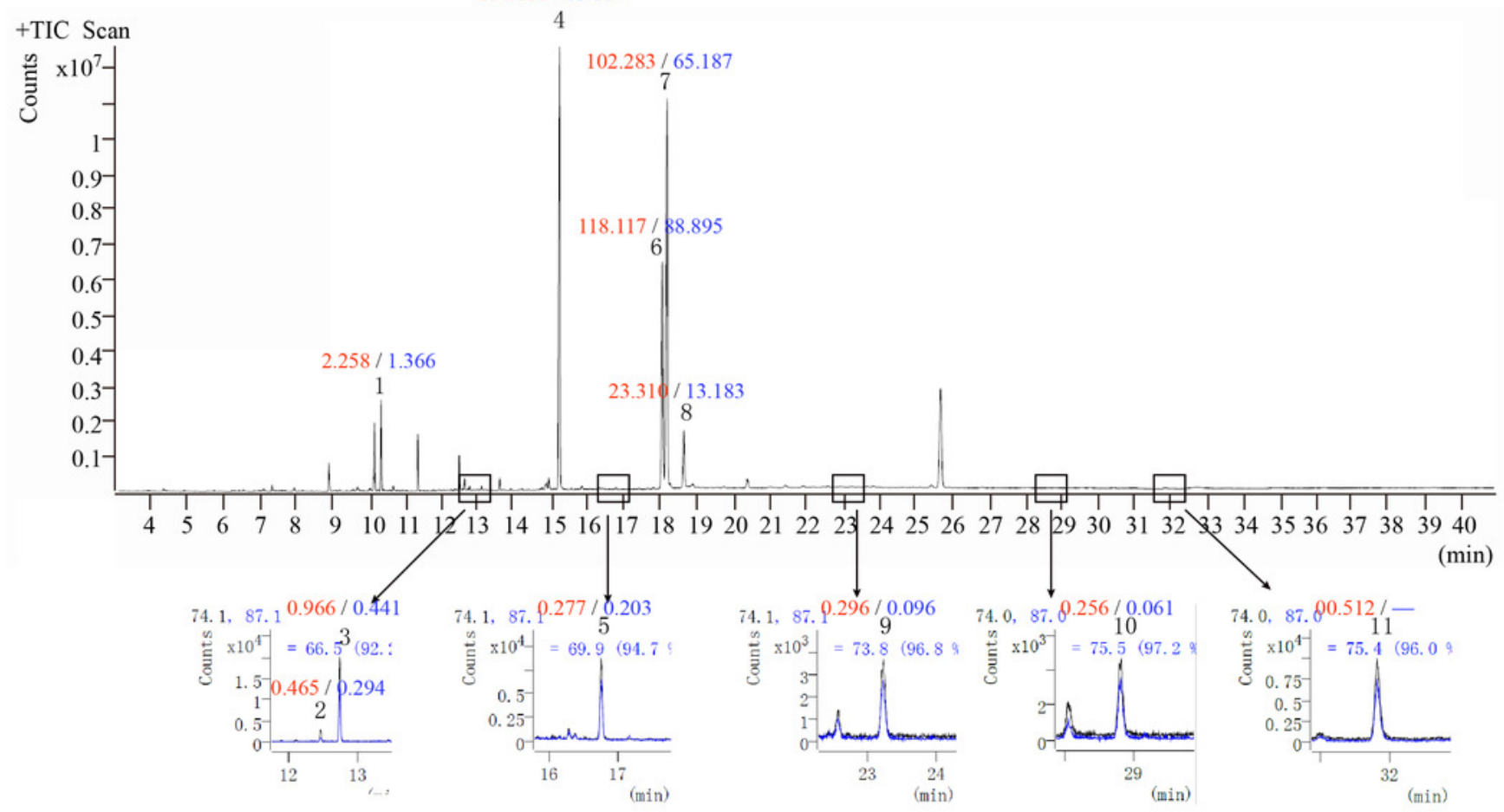

